\title{
Antenna Array Design for LOS-MIMO and Gigabit Ethernet Switch-Based Gbps Radio System
}

\author{
Chunhui Zhou, ${ }^{1}$ Xiang Chen, ${ }^{2}$ Xiujun Zhang, ${ }^{1}$ Shidong $Z_{h o u,}{ }^{1}$ Ming Zhao, ${ }^{1}$ and Jing Wang ${ }^{1}$ \\ ${ }^{1}$ Department of Electronic Engineering, Tsinghua National Laboratory for Information Science and Technology, Tsinghua University, \\ Beijing 100084, China \\ ${ }^{2}$ Aerospace Center, Tsinghua University, Beijing 100084, China
}

Correspondence should be addressed to Xiang Chen, chenxiang98@mails.tsinghua.edu.cn

Received 1 June 2012; Accepted 5 August 2012

Academic Editor: Sumei Sun

Copyright () 2012 Chunhui Zhou et al. This is an open access article distributed under the Creative Commons Attribution License, which permits unrestricted use, distribution, and reproduction in any medium, provided the original work is properly cited.

\begin{abstract}
The high spectrum efficiency of multiple-input multiple-output (MIMO) transmission traditionally depends on the high multiplexing gain in rich scattering environments, which will not always hold in the line-of-sight (LOS) environments, especially at higher microwave frequency band. In this paper, a novel antenna array design rule is proposed to guarantee full multiplexing gain for LOS-MIMO systems with one- or two-dimensional antenna arrays in LOS scenarios, and the strict perpendicular constraint is released in the two-dimensional case. The minimum antenna array area and the performance sensitivity to the area error are also obtained to guide the practical system design. Then, a demo MIMO-OFDM system with the designed square antenna array at $15 \mathrm{GHz}$ carrier is implemented on a novel Gigabit Ethernet (GE) switch-based software defined radio (SDR) platform, which combines the hardware accelerating units (HAUs) with the general-purpose processors (GPPs). The field evaluation results show that the system throughput and spectrum efficiency are greater than $1 \mathrm{Gbps}$ and $15 \mathrm{bps} / \mathrm{Hz}$, respectively. To the best of our knowledge, it is the first time to demonstrate the Gbps LOS-MIMO-OFDM system at such microwave bands in the world, which can be a successful design example for the next generation wireless backhaul or fixed wireless access.
\end{abstract}

\section{Introduction}

With the rapid development of modern cellular network and the explosion of mobile internet, the next generation cellular network is defined to provide higher user data rate than before, which requires the capacity of the backhaul network to improve accordingly, including the wireless backhaul in cases where it is hard to find the fiber. Traditional wireless backhaul is point to point with SISO antenna at microwave frequency. Cross polarization interference cancelation (XPIC) is often used to double the capacity with multiplexing gain of 2, but still not enough to catch up with the next generation backhaul.

There are some possible solutions to the problem. One is to use wider bandwidth at even higher frequency band [1]. Though the throughput of such system can be times of traditional microwave system, the modulation order is often limited due to the enlargement of phase noise. In [2], only QPSK is used for $60 \mathrm{GHz}$ frequency, which results in low spectrum efficiency. Another way is to increase the multiplexing gain by MIMO technology including antenna polarization $[3,4]$. Theoretically, the capacity can be increased unlimitedly with enough antennas when the channel state information is known at the transmitter, but it should be noted that the dimension of the antenna array will be a big problem for large antenna arrays. Some previous work [5-7] use these two technologies together, MIMO at millimeter wave frequency, to get both the multiplexing gain and wider bandwidth, but the spectrum efficiency is still low compared with MIMO system at microwave frequency.

In wireless backhaul, the channel is quasistatic LOS. Some previous works have come down to antenna array design for LOS-MIMO. Reference [8] analyzed the optimal condition for uniform linear array (ULA), and a $40-\mathrm{GHz}$ LOS-MIMO system is presented in [9] using ULA. The antenna arrays are extended to square and rectangle in [10, 11], but the two axes for the array should be perpendicular. This paper gives a more general rule to achieve optimal 
channel matrix (or maximum channel capacity) for oneand two-dimensional antenna arrays without perpendicular requirement. Also, the minimum area of antenna arrays is derived for optimal LOS-MIMO channel design based on our optimal rule. Further on, the impact of the array area error on channel capacity and channel matrix's condition number is analyzed to give a guideline for practical system design.

Based on the derived optimal condition of antenna array for LOS-MIMO, we design a high capacity fixed radio system using square antenna array symmetrical at both transmitter $\left(T_{x}\right)$ and receiver $\left(R_{x}\right)$. MIMO-OFDM, one of the most widely used technologies, is used as the basic transmission scheme, and the relatively more severe phase noise at microwave band is considered in channel estimation.

Usually, high capacity MIMO system needs complex signal processing which prefers a flexible, adaptable, programmable, and powerful SDR platform [12]. Generalpurpose processor- (GPP-) based platforms [13-15] have great adaptability and programmability, but the throughput is often limited due to the lack of real-time signal processing capacity. In contrast, SDR platforms based on hardware accelerating units (HAUs), for example, field programmable gate arrays (FPGAs), can provide strong ability for real-time signal processing but with relatively poor programmability. In this paper, a GE switch-based SDR platform for Gbps radio system is presented, which takes the advantages from both HAUs and GPPs to achieve good real-time processing performance with good adaptability and flexibility. Also, the HAU resources can be flexibly scheduled by choosing MIMO-OFDM as a transmission scheme and GE as a platform interface.

The field evaluation verifies our design rule for LOSMIMO antenna array, with the performance of up to Gbps throughput and $15 \mathrm{bps} / \mathrm{Hz}$ spectrum efficiency. Also, the spectrum efficiency can be doubled to $30 \mathrm{bps} / \mathrm{Hz}$ by crosspolarized antenna array without dimension increasing.

The remainder of this paper is organized as follows. We give the antenna array design rule for LOS-MIMO and sensitivity analysis in Section 2. The MIMO-OFDM-based LOS-MIMO Gbps radio system is presented in Section 3. A GE switch-based SDR platform and the implementation of the proposed Gbps system are given in Section 4. Field evaluation results are shown in Section 5. Finally, conclusions and discussions for future work are drawn in Section 6.

\section{Antenna Array Design}

2.1. Chanel Model and Capacity for LOS-MIMO. Consider a quasistatic LOS-MIMO channel with both antenna arrays at $T_{x}$ and $R_{x}$ side with the origins at $(0,0,0)$ and $\mathbf{R}_{0}=$ $(0,0, L)$ as shown in Figures 1 and 2 . The coordinates for $N T_{x}$ antennas and $M R_{x}$ antennas are $\mathbf{t}_{i}=\left(t_{i, x}, t_{i, y}, t_{i, z}\right), i=$ $1, \ldots, N$ and $\mathbf{r}_{i}+R_{0}=\left(r_{i, x}, r_{i, y}, r_{i, z}+L\right), i=1, \ldots, M$, where $L$ is the distance between $T_{x}$ and $R_{x}$. The distance for antenna pair $(i, j)$ is $d_{i j}=\left\|\mathbf{t}_{i}-\mathbf{r}_{j}-\mathbf{R}_{0}\right\|$. It is reasonable to assume that the distance between intra-array elements is much smaller than that between $T_{x}$ and $R_{x}$ array, so we have $d_{i j} \approx L$.
When pure LOS is considered, the channel from the $i$ th $T_{x}$ antenna to the $j$ th $R_{x}$ can be written as follows:

$$
H_{i j}=D\left(d_{i j}\right) \exp \left(-j \frac{2 \pi}{\lambda} d_{i j}\right) \approx D(L) \exp \left(-j \frac{2 \pi}{\lambda} d_{i j}\right),
$$

where $D(L)$ is the attenuation factor at transmission distance $L$. For a certain distance $L, D(L)$ is a constant for all antenna pairs, so it is omitted for the following capacity analysis.

Assuming $M \geq N$, the MIMO capacity under flat fading can be expressed as follows [16]:

$$
C=\sum_{i=1}^{N} \log _{2}\left(1+\lambda_{i} \mathrm{SNR}\right)
$$

where $\lambda_{i}=\operatorname{eig}\left(\mathbf{H}^{H} \mathbf{H}\right)$ is the eigenvalue of $\mathbf{H}^{H} \mathbf{H}$.

To optimize the channel capacity, the optimal channel matrix property is studied here. Let $\mathbf{W}=\mathbf{H}^{H} \mathbf{H}$, then

$$
\begin{aligned}
W_{k n} & =\sum_{m=1}^{M} H_{m k}^{H} H_{m n}=\sum_{m=1}^{M} \exp \left(j \frac{2 \pi}{\lambda}\left(d_{m k}-d_{m n}\right)\right) \\
& =\sum_{m=1}^{M} \exp \left(j \frac{2 \pi}{\lambda} \frac{d_{m k}^{2}-d_{m n}^{2}}{d_{m k}+d_{m n}}\right) \\
& =\sum_{m=1}^{M} \exp \left(j \frac{2 \pi}{\lambda} \frac{\left\langle\mathbf{t}_{k}, \mathbf{t}_{k}\right\rangle-\left\langle\mathbf{t}_{n}, \mathbf{t}_{n}\right\rangle+2\left\langle\mathbf{r}_{m}+\mathbf{R}_{0}, \mathbf{t}_{n}-\mathbf{t}_{k}\right\rangle}{d_{m k}+d_{m n}}\right),
\end{aligned}
$$

where $\langle\cdot, \cdot\rangle$ denotes the inner product of two vectors. Assuming $T_{x}$ antenna array is in $x-y$ plane, as depicted in Figures 1 and $2, t_{i, z}=0$, and $d_{i j}=L$, so we have

$$
W_{k n} \approx \exp \left(j \pi \frac{\left\langle\mathbf{t}_{k}, \mathbf{t}_{k}\right\rangle-\left\langle\mathbf{t}_{n}, \mathbf{t}_{n}\right\rangle}{\lambda L}\right) \sum_{m=1}^{m} \exp \left(j 2 \pi \frac{\left\langle\mathbf{r}_{m}, \mathbf{t}_{n}-\mathbf{t}_{k}\right\rangle}{\lambda L}\right) .
$$

The conditions for the channel to achieve its maximum are

$$
\begin{gathered}
C=C_{\max } \Longleftrightarrow \\
\forall k \neq n, \quad W_{k n}=0 \Longleftrightarrow \\
\forall k \neq n, \quad \sum_{m=1}^{M} \exp \left(j \pi \frac{\left\langle\mathbf{r}_{m}, \mathbf{t}_{n}-\mathbf{t}_{k}\right\rangle}{\lambda L}\right)=0 .
\end{gathered}
$$

Equation (5) shows that to make the $T_{x}$ and $R_{x}$ array parallel and perpendicular to the transmission direction $(z$ axis) can reduce the size of antenna array, but it is not a necessary condition to achieve optimal capacity.

2.2. One-Dimensional Linear Array. The one-dimensional linear array case is shown in Figure 1 with equal spacing at both $T_{x}$ and $R_{x}$ side, where $\mathbf{v}_{t}\left(\mathbf{v}_{r}\right)$ is the $T_{x}\left(R_{X}\right)$ vector with amplitude of $T_{x}\left(R_{x}\right)$ array spacing and orientation of $T_{x}\left(R_{x}\right)$ 


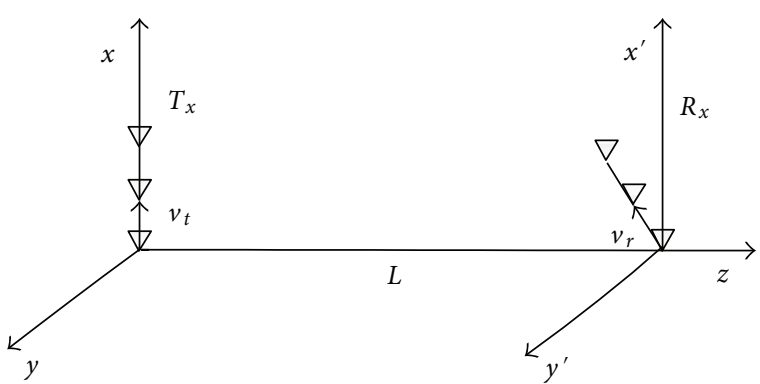

FIGURE 1: Linear antenna array with equal spacing.

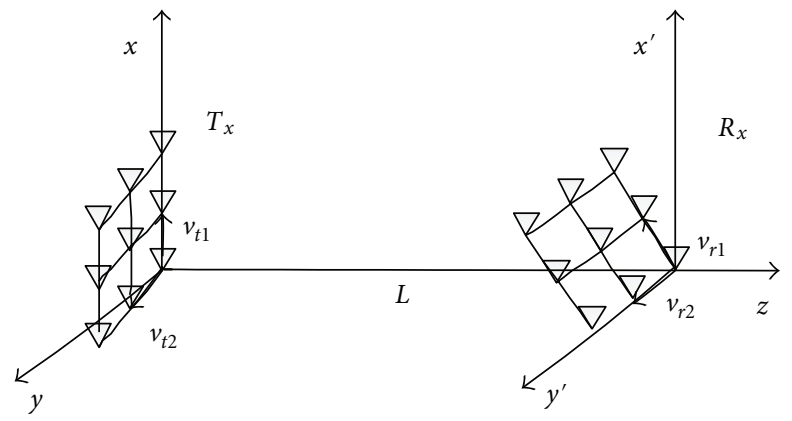

FIgURE 2: Two-dimensional antenna array.

array direction. Then, we have $\mathbf{t}_{n}-\mathbf{t}_{k}=(n-k) \mathbf{v}_{t}$, and (5) is reduced to

$$
\forall n \in\{ \pm 1, \ldots, \pm N-1\}, \sum_{m=1}^{M} \exp \left(j 2 \pi n \frac{\left\langle\mathbf{r}_{m}, \mathbf{v}_{t}\right\rangle}{\lambda L}\right)=0 .
$$

One sufficient condition for (6) can be found as follows:

$$
\frac{\left\langle\mathbf{r}_{m}, \mathbf{v}_{t}\right\rangle}{\lambda L}=\frac{(m-1) p}{N},
$$

where $\operatorname{gcd}(p, N)=1, M=k N, p, k \in \mathbb{Z}^{+}$.

With the validity of (7), the sum in (6) is the sum of all the $(N / \operatorname{gcd}(n, N))$-th complex root of 1 , which should be 0 . Here, $\operatorname{gcd}(\cdot, \cdot)$ denotes the greatest common divisor for two positive integers.

Now let us consider the most compact linear array design with MIMO multiplexing gain of $N$. Let $p=1$ and $M=N$, we get $\left\langle\mathbf{v}_{\mathbf{r}}, \mathbf{v}_{\mathbf{t}}\right\rangle=\lambda L / N$. It is obvious to set the two arrays parallel to make the minimum antenna separation product (ASP) [4] $\left\|\mathbf{v}_{\mathbf{r}}\right\| \cdot\left\|\mathbf{v}_{\mathbf{t}}\right\|=\lambda L / N$. Let $R_{t}$ and $R_{r}$ be the lengths of antenna arrays at $T_{x}$ and $R_{x}$, then we have the optimal condition:

$$
R_{t} R_{r}=\frac{(N-1)^{2}}{N} \lambda L .
$$

In case of equal size at $T_{x}$ and $R_{x}$, from (8) we get the optimal array length as follows:

$$
R_{t}=R_{r}=(N-1) \sqrt{\frac{\lambda L}{N}} .
$$

2.3. Two-Dimensional Array. The two-dimensional antenna array case is shown in Figure 2, where $T_{x}$ array is placed at $x-y$ plane with $N_{1} \times N_{2}$ elements and $R_{x}$ array at $x^{\prime}-y^{\prime}$ plane with $M_{1} \times M_{2}$ elements. And the array elements are renumbered as follows:

$$
\begin{aligned}
\mathbf{t}_{n_{1}, n_{2}} & =\left(n_{1}-1\right) \mathbf{v}_{t_{1}}+\left(n_{2}-1\right) \mathbf{v}_{t_{2}}, \\
\mathbf{r}_{m_{1}, m_{2}} & =\left(m_{1}-1\right) \mathbf{v}_{r_{1}}+\left(m_{2}-1\right) \mathbf{v}_{r_{2}},
\end{aligned}
$$

where $\mathbf{v}_{t_{1}}, \mathbf{v}_{t_{2}}$ and $\mathbf{v}_{r_{1}}, \mathbf{v}_{r_{2}}$ are the two-dimensional extensions of one-dimensional $\mathbf{v}_{t}, \mathbf{v}_{r}$ with the similar definition. Note that when $\mathbf{t}_{n_{1}, n_{2}}-\mathbf{t}_{k_{1}, k_{2}}=\left(n_{1}-k_{1}\right) \mathbf{v}_{t_{1}}+\left(n_{2}-k_{2}\right) \mathbf{v}_{t_{2}}$, (5) can be rewritten as follows:

$$
\begin{gathered}
\forall n_{i} \in\left\{0, \pm 1, \ldots, \pm N_{i}-1\right\}, \quad i=1,2 \text {, and } n_{1} n_{2} \neq 0, \\
\sum_{m_{1}=0}^{M_{1}-1} \exp \left(j 2 \pi n_{1} m_{1} \frac{\left\langle\mathbf{v}_{\mathbf{t}_{1}}, \mathbf{v}_{\mathbf{r}_{1}}\right\rangle}{\lambda L}\right) \exp \left(j 2 \pi n_{2} m_{1} \frac{\left\langle\mathbf{v}_{t_{1}}, \mathbf{v}_{r_{2}}\right\rangle}{\lambda L}\right) \\
\cdot \sum_{m_{2}=0}^{M_{2}-1} \exp \left(j 2 \pi n_{1} m_{2} \frac{\mathbf{v}_{t_{2}}, \mathbf{v}_{r_{1}}}{\lambda L}\right) \exp \left(j 2 \pi n_{2} m_{2} \frac{\mathbf{v}_{t_{2}}, \mathbf{v}_{r_{2}}}{\lambda L}\right)=0 .
\end{gathered}
$$

Set $n_{1}=0, n_{2}=0$, respectively, we get the necessary conditions:

$$
\begin{aligned}
& \forall n_{i} \in\left\{1, \ldots, N_{i}-1\right\}, \quad i=1,2, \\
& \sum_{m_{1}=0}^{M_{1}-1} \exp \left(j 2 \pi n_{i} m_{1} \frac{\left\langle\mathbf{v}_{t_{1}}, \mathbf{v}_{r_{i}}\right\rangle}{\lambda L}\right) \sum_{m_{2}=0}^{M_{2}-1} \exp \left(j 2 \pi n_{i} m_{2} \frac{\left\langle\mathbf{v}_{t_{2}}, \mathbf{v}_{r_{i}}\right\rangle}{\lambda L}\right) \\
& =0 .
\end{aligned}
$$

When $\left\langle\mathbf{v}_{t_{1}}, \mathbf{v}_{r_{2}}\right\rangle=\left\langle\mathbf{v}_{t_{2}}, \mathbf{v}_{r_{1}}\right\rangle=0,\left\langle\mathbf{v}_{t_{2}}, \mathbf{v}_{r_{2}}\right\rangle\left\langle\mathbf{v}_{t_{1}}, \mathbf{v}_{r_{1}}\right\rangle \neq 0$ (or $\left.\left\langle\mathbf{v}_{t_{2}}, \mathbf{v}_{r_{2}}\right\rangle=\left\langle\mathbf{v}_{t_{1}}, \mathbf{v}_{r_{1}}\right\rangle=0,\left\langle\mathbf{v}_{t_{1}}, \mathbf{v}_{r_{2}}\right\rangle\left\langle\mathbf{v}_{t_{2}}, \mathbf{v}_{r_{1}}\right\rangle \neq 0\right)$, (12) can be further simplified to

$$
\begin{gathered}
\forall n_{i} \in\left\{1, \ldots, N_{i}-1\right\}, \\
\sum_{m_{i}=0}^{M_{i}-1} \exp \left(j 2 \pi n_{i} m_{i} \frac{\left\langle\mathbf{v}_{t i}, \mathbf{v}_{r i}\right\rangle}{\lambda L}\right)=0, \quad i=1,2 .
\end{gathered}
$$

Equation (13) is similar to the one-dimensional case. The one sufficient condition for two-dimensional array case can be concluded as follows: (1) the first dimension of $T_{x} / R_{x}$ and second dimension of $T_{x} / R_{x}$ satisfy optimal linear array condition respectively, (2) the first dimension of $T_{x}$ is orthogonal to the second dimension of $R_{x}$, and so as the second dimension of $T_{x}$ and the first of $R_{x}$.

Consider the most compact array design and equal number of elements at $T_{x}$ and $R_{x}, p_{i}=1, M_{i}=N_{i}$, the array area satisfies

$$
\frac{S_{t} S_{r}}{\lambda L}=\frac{\left(N_{1}-1\right)^{2}\left(N_{2}-1\right)^{2}}{N_{1} N_{2}},
$$

where $S_{t}$ and $S_{r}$ are the array area of $T_{x}$ and $R_{x}$, respectively. In case of symmetrical array at $T_{x}$ and $R_{x}$, the array area yields to $S_{t}=S_{r}=\left(\left(N_{1}-1\right)\left(N_{2}-1\right) / \sqrt{N_{1} N_{2}}\right) \lambda L$. 
$15 \mathrm{GHz}, L=2 \mathrm{~km}, \mathrm{SNR}=30 \mathrm{~dB}$, equal array size at $T_{x}$ and $R_{x}$

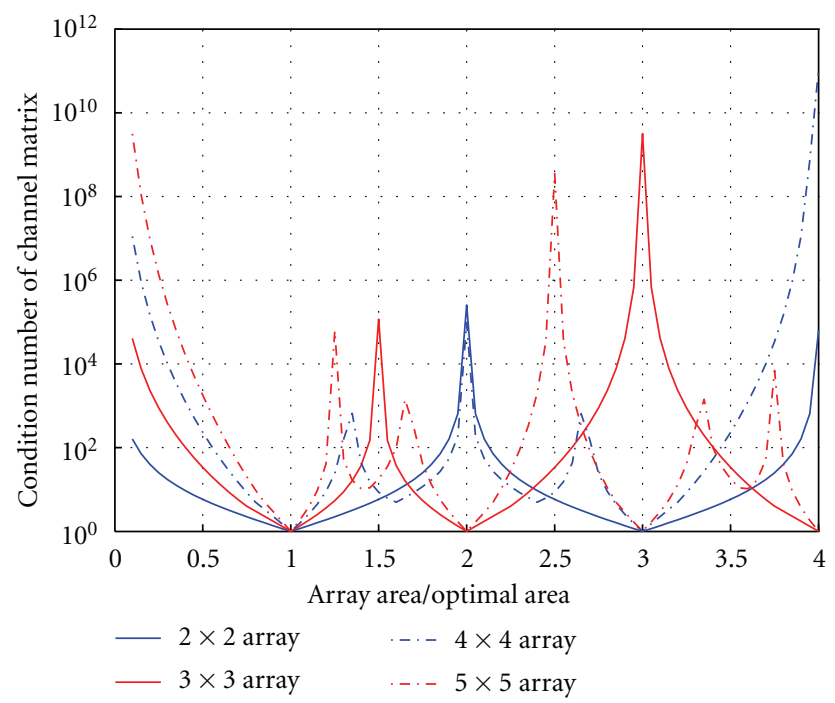

Figure 3: Channel condition number versus antenna array area.

$15 \mathrm{GHz}, L=2 \mathrm{~km}, \mathrm{SNR}=30 \mathrm{~dB}$, equal array size at $T_{x}$ and $R_{x}$

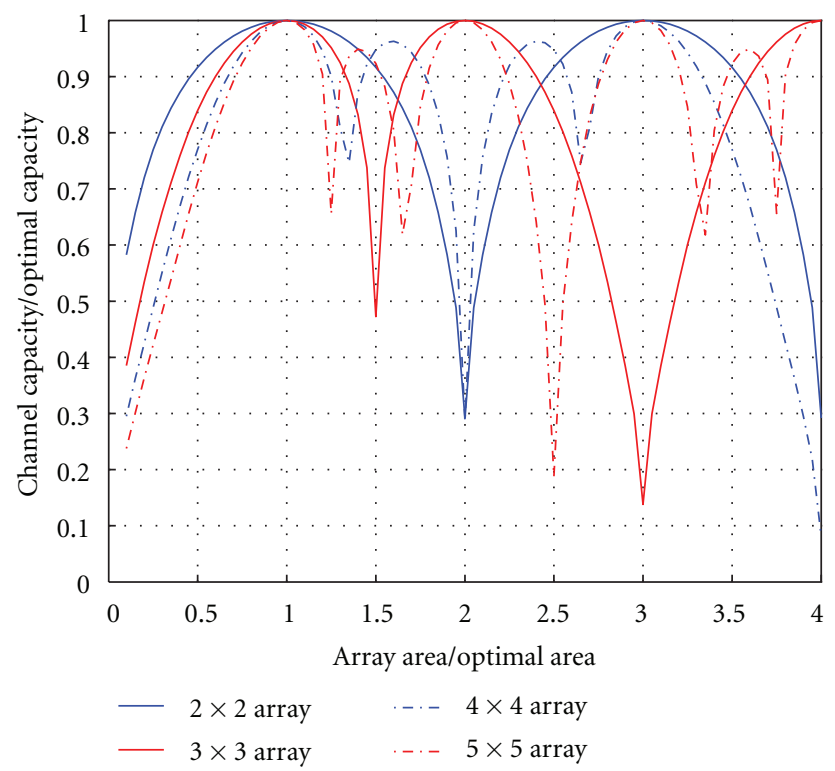

FIGURE 4: Channel capacity versus antenna array area.

From (14), the multiplexing gain is $S_{t} S_{r} /(\lambda L)^{2}$ at large $N_{i}$, which is in accordance with the result in [10]. The result here is more general than in [10], which there is no perpendicular requirement for the two-dimensional array itself, so it leaves the flexible shape choice, from any parallelogram, to the system designer.

As a practical example, Table 1 shows the minimum array area for some typical array configurations when symmetrical
$15 \mathrm{GHz}, L=2 \mathrm{~km}, 4 \times 4$ array, equal size at $T_{x}$ and $R_{x}$

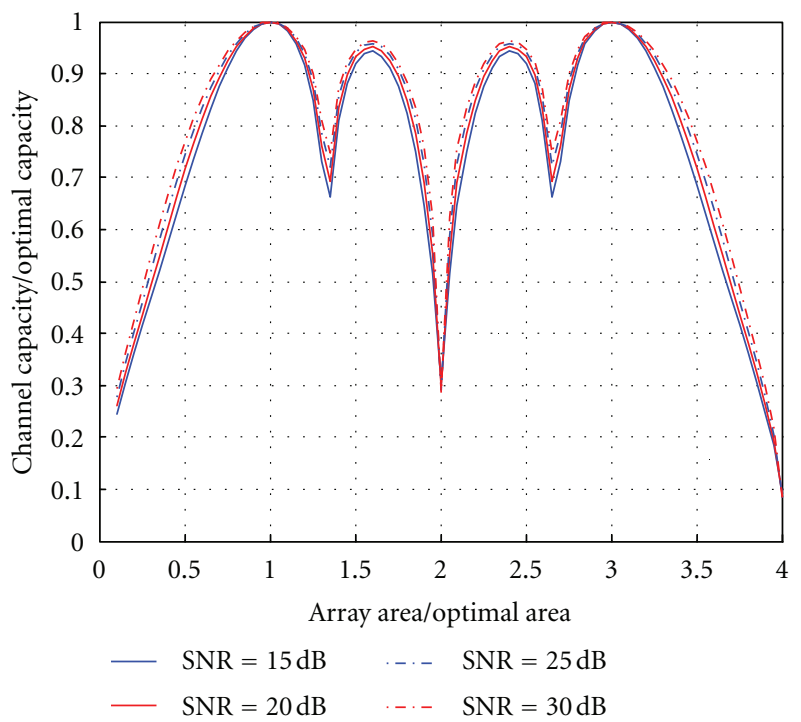

Figure 5: Channel capacity versus antenna array area.

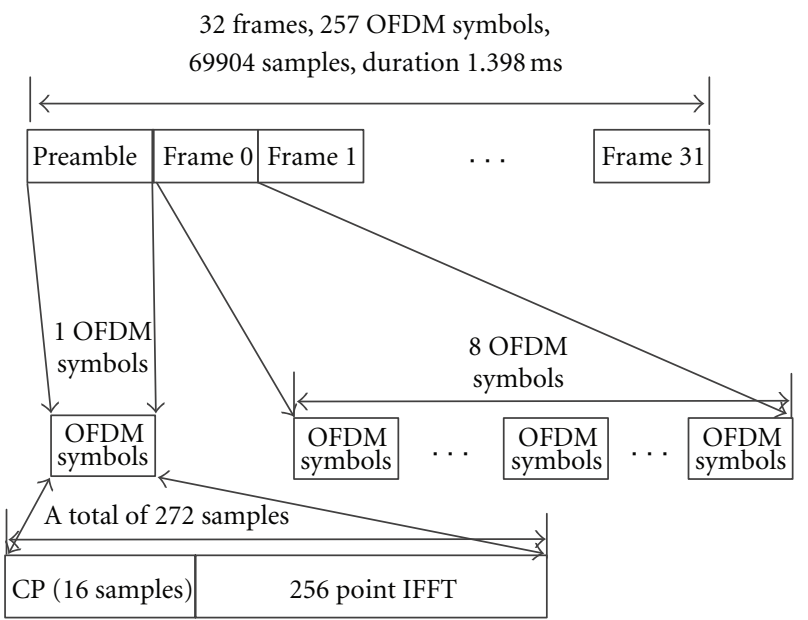

Figure 6: Frame structure of one super frame.

array is used. The frequency and transmission distance are $15 \mathrm{GHz}$ and $2 \mathrm{~km}$, respectively.

2.4. Sensitivity Analysis for Array Area. From previous sections, we know the optimal parameters, but it is reasonable to encounter some deviations from installation and manufacturing in practice, especially for the antenna array area. It is necessary to do the sensitivity analysis to see the robustness of the derived rule. For high capacity fixed wireless access application, we generally focus on high SNR regime. Different SNR conditions are compared in the sensitivity analysis of array area.

As for performance measurement, the condition number of channel matrix and channel capacity are adopted. The condition number of channel matrix $\mathbf{H}$ is denoted as $\operatorname{cond}(\mathbf{H})$, which is the ratio of the maximum and minimum 
TABLE 1: Minimum array area for different configurations.

\begin{tabular}{lccccccccc}
\hline Antenna array & $2 \times 2$ & $2 \times 3$ & $3 \times 3$ & $2 \times 5$ & $2 \times 7$ & $3 \times 5$ & $3 \times 7$ & $2 \times 11$ & $5 \times 5$ \\
\hline Minimum area $\left(\mathrm{m}^{2}\right)$ & 20 & 32.7 & 53.3 & 50.6 & 64.1 & 82.6 & 104.7 & 85.3 & 128 \\
\hline
\end{tabular}

singular values of $\mathbf{H}$. In LOS-MIMO case, the optimal channel corresponds to $\operatorname{cond}(\mathbf{H})=1$, and the large condition number leads to lower channel capacity.

The simulation results of sensitivity analysis for the condition number and capacity due to array area deviation are shown in Figures 3 and 4 under the conditions of $15 \mathrm{GHz}$ frequency band, $L=2 \mathrm{~km}, \mathrm{SNR}=30 \mathrm{~dB}$, and equal to array size at $T_{x}$ and $R_{x}$. The ratio of actual area to the optimal minimum area is used as the analyzing parameter and different array configurations are considered. In Figure 5, the same performance items are shown with different $S N R$ values from $15 \mathrm{~dB}$ to $30 \mathrm{~dB}$ for typical $4 \times 4$ array.

The results show that the condition number and capacity are insensitive to area deviation for most array configurations, but the performance degradation increases with more antenna elements. Take $90 \%$ of optimal capacity as a threshold, the tolerable ranges of area deviation ratio for $2 \times 2$ and $5 \times 5$ arrays are $(0.46,1.54)$ and $(0.71,1.2)$, respectively at $\mathrm{SNR}=30 \mathrm{~dB}$. From Figure 5, it can be seen that the area tolerance ranges are very close for different SNRs, which means that the proposed design rule is also not sensitive to SNR. Another interesting finding is that for $2 \times 2$ and $4 \times 4$ array, the optimal capacity can also be achieved when actual area is 3 times of the minimum optimal ones, and for $3 \times 3$ and $5 \times 5,2$ times of minimum optimal areas are still optimal. These are due to the relatively prime property of $p$ and $N$ in these cases as in (7).

\section{MIMO-OFDM-Based LOS-MIMO Gbps System}

MIMO systems provide an additional spatial dimension for wireless communications and yield a degree-of-freedom gain. These additional degrees of freedom lead to obvious capacity increase: for a system with $N$ transmit and receive antennas, respectively, the capacity increase is proportional to $N$. Meanwhile, orthogonal frequency division multiplexing (OFDM) modulates the information on parallel subcarriers in the frequency domain. It has a distinct advantage in antifading ability than traditional single-carrier technologies.

Therefore, MIMO-OFDM [17, 18], which combines MIMO and OFDM technologies, is considered as the physical layer scheme in our Gbps radio system, due to its distinct advantages in many aspects of system performance such as system capacity, spectral efficiency, and antifading ability.

3.1. Main Parameters. To achieve 1 Gbps throughput, the system occupies $2 \times 33 \mathrm{MHz}$ bandwidth at $15 \mathrm{GHz}$ frequency band. For each $33 \mathrm{MHz}$ bandwidth, a $4 \times 4$ MIMO subsystem
TABle 2: General link level parameters.

\begin{tabular}{lc}
\hline Parameters & Value \\
\hline$T_{x} / R_{x}$ antennas & $4 \times 4$ \\
Carrier frequency $(\mathrm{GHz})$ & $14.417 / 14.483$ \\
Bandwidth $(\mathrm{MHz})$ & 33 for each set \\
Sample rate $(\mathrm{Msps})$ & 40.96 \\
Channel coding & $(7680,6400)$ QC-LDPC \\
Modulation & 64QAM \\
Channel condition & Indoor $(50$ m LOS environments $)$ \\
\hline
\end{tabular}

TABLE 3: OFDM parameters.

\begin{tabular}{lc}
\hline Parameters & Value \\
\hline FFT size & 256 \\
Modulated subcarriers & 168 \\
Subcarrier bandwidth $(\mathrm{KHz})$ & 160 \\
OFDM symbol length (us) & 6.64 \\
CP length (us) & 0.39 \\
OFDM symbols per data frame & 8 \\
Pilot/data OFDM symbols & $1 / 64$ \\
\hline
\end{tabular}

is used. The parameters of each $4 \times 4$ MIMO-OFDM set are given in Tables 2 and 3.

The system's frame structure is shown in Figure 6. One super frame consists of one preamble and 32 data frames.

A super frame starts with a preamble, which has the same duration as an OFDM symbol (6.64 us) and is used for timing synchronization, channel estimation, and phase noise correction. The Chu sequence [19] is introduced in the system as preamble for its constant modulus and cyclostationarity.

There are 32 data frames in one super frame, and each of them includes 8 OFDM symbols. An OFDM symbol consists of a cyclic prefix and 256 points IFFT. 168 of 256 subcarriers are used for each OFDM symbol.

\subsection{Algorithm Design}

3.2.1. Preamble. To facilitate quick synchronization, the data packet is preceded with a known sequence (the preamble). The preamble is carefully designed to provide enough information for a good packet detection, phase noise estimation, and channel estimation. The Chu sequence is introduced in the system as preamble for its constant modulus and cyclostationarity. It is given by [19]

$$
p(k)=\left\{\begin{array}{ll}
e^{j \pi\left(M / N_{p}\right) k^{2}}, & N_{p} \text { is even, } \\
e^{j \pi\left(M / N_{p}\right) k(k+1)}, & N_{p} \text { is odd, }
\end{array} \quad k=0,1, \ldots, N_{p}-1,\right.
$$




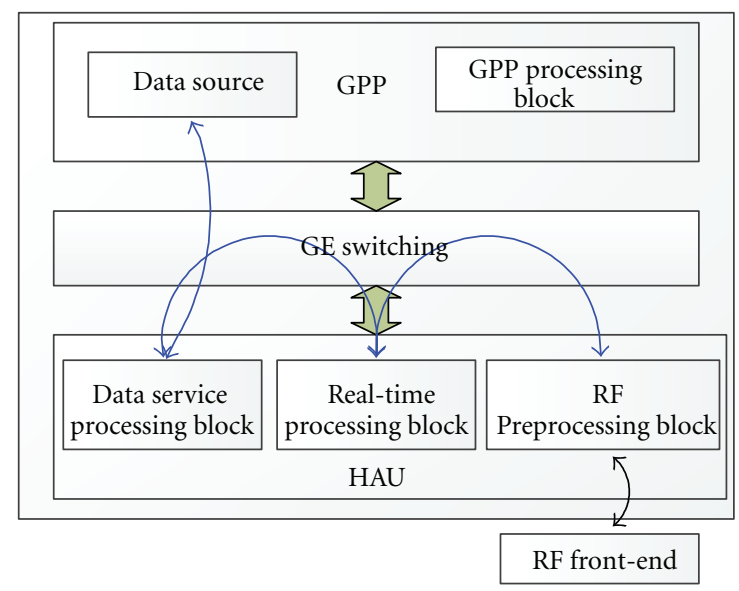

FIgURE 7: GE-based SDR platform.

where $N_{p}$ is the length of the sequence and $M$, the sequence index, is relatively prime to $N_{p}$.

To estimate the MIMO channel, it is important that the subchannels from the different $T_{x}$ antennas to every $R_{x}$ antenna can be uniquely identified. To achieve this the preambles on the different $T_{x}$ antennas should be orthogonal. Besides, to perform the phase noise correction, a few subcarriers should be reserved in OFDM symbols not only in preamble frames but also the data frames, in which the receiver could estimate the phase rotation between the data frames and preamble frames.

3.2.2. Channel Estimation. The channel response can be estimated using the known training symbols within the preamble. When the timing is recovered correctly, we know which received samples correspond to the training part for channel estimation. More precisely, we know exactly which part of the received preamble is sent by transmit antenna $m$. The 4 transmit antennas share the subcarriers in preamble orthogonally by means of frequency division.

With the preamble, we can estimate the channel response values $\mathbf{h}_{m}\left[k_{1}\right], \ldots, \mathbf{h}_{m}\left[k_{n}\right]$, where $m$ is transmit antenna number, and $k$ denotes the location of the subcarriers sent by transmit antenna $m$. As only one channel value in every four subcarriers can be obtained directly from the training symbols, the values on the other subcarriers are estimated by a linear interpolation scheme based on minimum-meansquare-error (MMSE) criteria.

3.2.3. Phase Noise Correction. As mentioned in Section 3.2.1, the receiver could estimate the phase rotation between the data frames and preamble frames with the reserved subcarriers in each OFDM symbol. The phase noise matrix is denoted by

$$
\boldsymbol{\Phi}=\left[\begin{array}{cccc}
r_{1} t_{1} & r_{1} t_{2} & \cdots & r_{1} t_{M} \\
r_{2} t_{1} & r_{2} t_{2} & \cdots & r_{2} t_{M} \\
\vdots & \vdots & \ddots & \vdots \\
r_{N} t_{1} & r_{N} t_{2} & \cdots & r_{N} t_{M}
\end{array}\right]
$$

where $r_{i}=e^{\varphi_{i}}$ and $t_{i}=e^{\theta_{i}}$, where $\varphi_{i}$ and $\theta_{i}$ denote the phase noise on $i$ th receive and transmit antenna, respectively. The transmit phase noise vector is defined as $\overline{\boldsymbol{\theta}}=\left[\begin{array}{llll}1 & t_{2} / t_{1} & \cdots & t_{M} / t_{1}\end{array}\right] \cdot \overline{\boldsymbol{\theta}}$ can be obtained by

$$
\overline{\boldsymbol{\theta}}=\frac{1}{N}\left[\begin{array}{l}
1 \\
1 \\
\cdot \\
\cdot \\
1
\end{array}\right]^{T}\left[\begin{array}{lllll}
1 & & & \\
1 & & & & \\
1 & \boldsymbol{\Phi}_{2} \cdot / \boldsymbol{\Phi}_{1} & \boldsymbol{\Phi}_{3} \cdot / \boldsymbol{\Phi}_{1} & \cdots & \boldsymbol{\Phi}_{M} \cdot / \boldsymbol{\Phi}_{1} \\
1 & & &
\end{array}\right],
$$

where $\boldsymbol{\Phi}_{i}$ is the $i$ th column of the matrix $\boldsymbol{\Phi}$. Similarly, the receive phase noise vector is defined as $\bar{\varphi}=$ $\left[\begin{array}{llll}r_{1} t_{1} & r_{2} t_{1} & \cdots & r_{N} t_{1}\end{array}\right]$, which can be estimated by

$$
\overline{\boldsymbol{\varphi}}=\frac{1}{M}\left[\begin{array}{c}
\boldsymbol{\Phi}_{0}^{r} \cdot \overline{\boldsymbol{\theta}} \\
\boldsymbol{\Phi}_{1}^{r} \cdot / \overline{\boldsymbol{\theta}} \\
\cdot \\
\cdot \\
\boldsymbol{\Phi}_{N}^{r} \cdot \overline{/ \boldsymbol{\theta}}
\end{array}\right]\left[\begin{array}{c}
1 \\
1 \\
\cdot \\
\cdot \\
1
\end{array}\right],
$$

where the $\boldsymbol{\Phi}_{i}^{r}$ is the $i$ th row of $\boldsymbol{\Phi}$. Once the $\overline{\boldsymbol{\theta}}$ and $\overline{\boldsymbol{\varphi}}$ are obtained, the channel matrix for current OFDM symbol is given by

$$
\overline{\mathbf{H}}=\operatorname{diag}(\overline{\boldsymbol{\varphi}}) \mathbf{H} \operatorname{diag}(\overline{\boldsymbol{\theta}}) .
$$

\section{GE-Based SDR Platform and Implementation of Gbps System}

4.1. Platform Architecture. According to the OFDM theory, the whole transmission band of OFDM signals can be divided into several subbands, which can be processed independently. The multiband capability of the OFDM makes the design of broadband system much more flexible. GE interfaces are involved to connect multiple processing units and RF front-ends. Based on GE switching, we establish a high-throughput pipeline for data stream as well as a lowlatency path for the control signals switched between each unit in our SDR platform. A distributed processing strategy is adopted to get high-efficiency parallel computing ability and to reduce the requirements of computation capacity for each processing unit. The platform takes the advantages from both HAUs and GPPs to achieve good real-time processing performance with good adaptability and flexibility. The detailed architecture of the GE switch-based SDR platform is illustrated in Figure 7.

This platform can be divided into the following four function blocks: data services processing block, real-time processing block, radio frequency (RF) preprocessing block, and GPP processing block. Taking the transmitter side, for example, we can show the data switching process on the platform as follows.

The user data stream is firstly transformed into fixedlength packets by data service processing block. With GE switching, data packets are distributed to different sub-bands processing units and recollected after frequency-domain processing (e.g., channel coding, modulation, subcarrier 


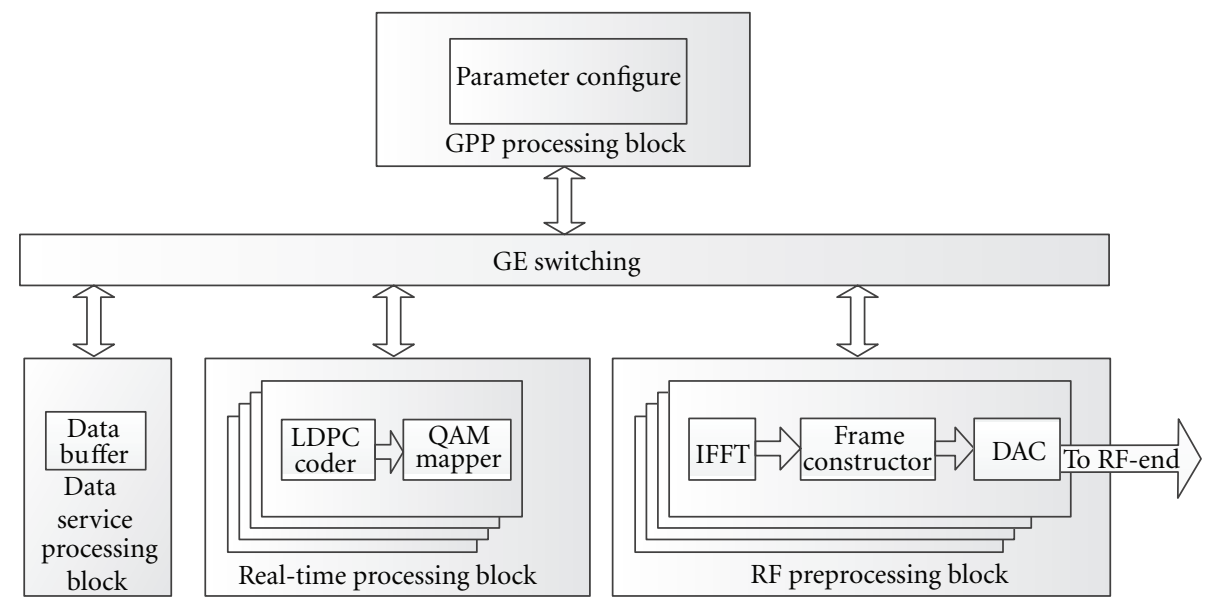

FIgURE 8: Transmitter structure.

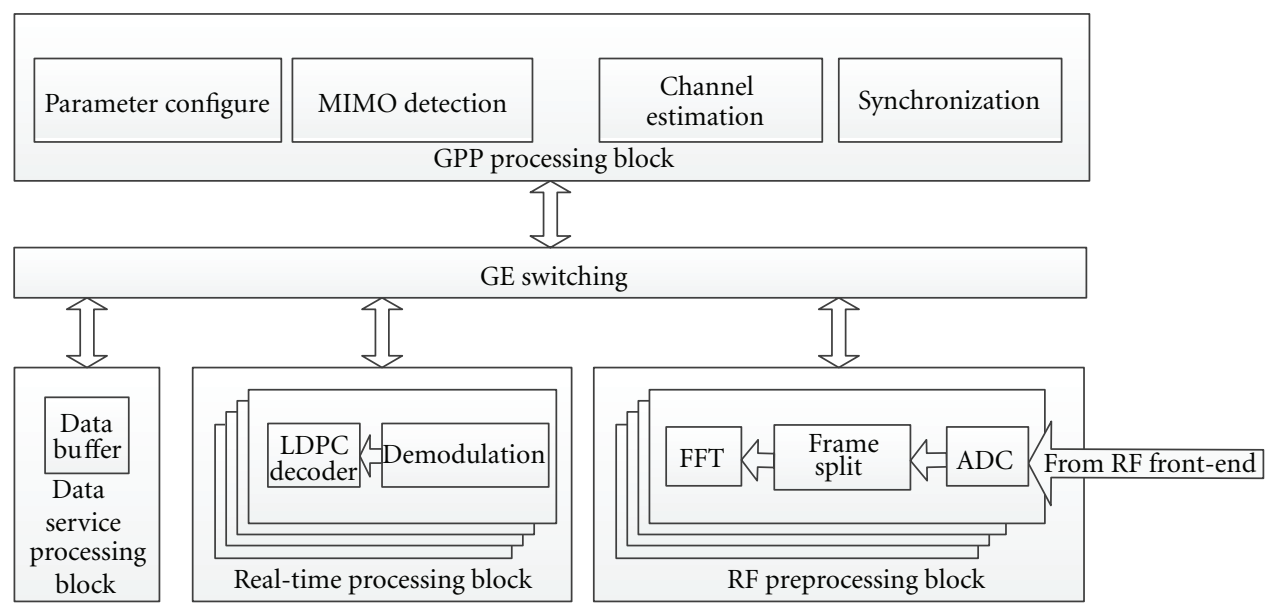

Figure 9: Receiver structure.

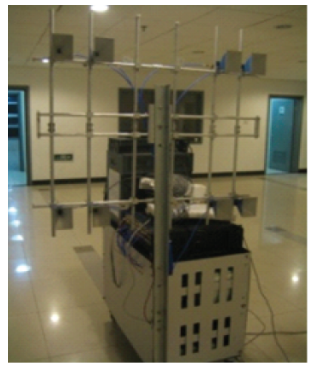

Transmitter
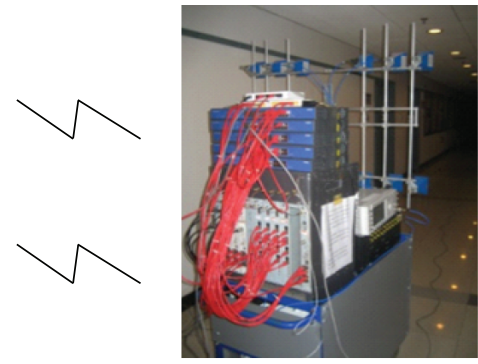

Receiver
FIGURE 10: Transmitter and receiver on the GE SR platform.

mapping, and precoding) in each real-time processing block. Then, the data packets in the frequency domain are distributed to each of the RF preprocessing block (performing IFFT, inserting of CP, etc.) through GE switching. Finally, after time-domain processing the data streams are transmitted to the RF front-ends.
The basic functional partition for the receiver side is similar to the above transmitter side. However, most complex computation processes, such as channel estimation, timing and frequency synchronization, and matrix decompositions for MIMO detecting and precoding, have been allocated to GPP processing block. The left function for the receiver is only channel decoding module in real-time processing block for HAUs.

On the other hand, GE interface is a powerful debugging tool for system developers. It is convenient to get data samples from any in-out port in this platform by devices supporting GE.

4.2. Implementation of Gbps System on GE SDR Platform. Based on the above antenna array design and MIMO-OFDM scheme for LOS-MIMO, the Gbps system is implemented on the GE switch-based SDR platform, utilizing its flexibility, programmability, and real-time processing ability. Though the traffic data processing and RF front-end are important 
modules of the system, only the transmitter and receiver path are discussed in detail.

The transmitter structure and the process partition are shown in Figure 8.

User application data first goes through the data service processing block and is multiplexed into constant bit rate stream and mapped to the standard GE packet for switching.

Then, the data stream is distributed to 4 real-time processing units in real-time processing block, corresponding to four sub-bands, where the data stream goes through a QC-LDPC encoder and is mapped to 64QAM symbols. After that, a multiantenna multiplexing module splits the symbols into four independent parallel data streams, and each one is corresponding to a transmitting antenna. Similarly, the data stream is passed to RF preprocessing block in the form of GE MAC packets.

In each RF preprocessing unit corresponding to each transmitting antenna, data samples in the same frame are collected from every subband to calculate 256-point IFFT. Then, the CP is inserted to every OFDM symbols. Finally, a preamble which includes training sequences is added to every super frame before the final $T_{X}$ signal is upconverted to the RF front-end and transmitted.

The GPP processing block can configure the parameters for all the HAU blocks.

Compared with the transmitter, GPP processing block plays a more important role in the receiver. Although the real-time processing capacity of GPPs cannot match the needs of Gbps transmission, the algorithms working on lowrate samples, such as channel estimation for slow fading channels, timing and frequency synchronization, and matrix decompositions for MIMO detecting and precoding, could be implemented on GPPs for their great flexibility and programmability. The receiver structure and the process partition are shown in Figure 9.

First, four receive antennas are used for each set of MIMO-OFDM system and low-rate samples of received data go through the GE interface from RF preprocessing block to GPP processing block, where a correlation method in time domain is used to achieve time synchronization and frequency offset estimation, then GPP processing block informs the results to RF preprocessing block. In doing so, RF preprocessing units for every antenna can identify from the sampled sequences the starting points of $S / P$ conversion of OFDM data symbols. Then, frequency-domain OFDM symbols can be obtained by (1) correcting frequency offset, (2) removing the $\mathrm{CP}$, and executing FFT algorithms.

The frequency-domain OFDM symbol in preamble is sent to GPP processing block, where channel estimation is taking place. Then, the MIMO detecting matrix and phase noise for every subcarrier can be estimated in frequencydomain. The result is transmitted to real-time processing block. In order to meet the $1 \mathrm{G}$ transmission data rate, four real-time processing units are used. On each unit, data samples of each sub-band in the frequency domain perform phase noise correction and MIMO detection, then QCLDPC decoding algorithms are executed. Finally the user application data packet is recovered from decoded data block in data service processing block.

\section{Field Evaluation of LOS-MIMO Gbps System}

In this section, we will give the field evaluation results of the end-to-end Gbps transmission performance. In our MIMOOFDM demonstration system over the GE switch-based SDR platform, a PC with dual-core CPU operating at $1.6 \mathrm{GHz}$ is used as the GPP at each side of transmitter and receiver. For each HAU, two Altera Cyclone 3C80 FPGA chips are used for computation, combined with one GE PHY chip (BCM5464) to support $4 \mathrm{GE}$ interfaces.

A series of experiments and field trials of our Gbps demonstration system has been carried out at the FIT Building in Tsinghua University, China. As mentioned in Section 3 , the demonstration radio system consists of two sets of $4 \times 4$ MIMO-OFDM subsystems. Figure 10 shows the transmitter and receiver, respectively.

The field tests were done in indoor environments with the transmission distance over $50 \mathrm{~m}$. The SNR and BER performance for different locations are depicted in Table 4, where the $T_{X}$ and $R_{X}$ logo are coordinates for $T_{X}$ and $R_{X}$ positions along the corridor. In the in-building corridor scenario, there exists visible 2-path effect due to reflection of the walls at each end of the corridor. The 2nd path delay and relative power are given in Table 4, which suffer some variation for different locations. The 2 nd path delay is decided mainly by the length of the corridor, and the relative power is below $20 \mathrm{dBc}$ for most cases. The results show that the proposed and implemented system works well under such 2-path scenario.

The maximum achievable information bit rates are calculated as $1.171 \mathrm{Gbps}=2$ (two sets of subsystems) $\times$ 32 (frames per super frame) $\times 8$ (OFDM symbols per frame) $\times 160$ (subcarriers per OFDM symbols) $\times 6$ (index for 64-QAM modulation) $\times 4$ (numbers of transmitting antennas) $\times 5 / 6($ LDPC coding rate)/0.001398 (super frame duration). Table 5 shows the field test results of the peak data transmission throughput of the Gbps platform by different methods, where Netperf is a PC-based network benchmark tool. We also use self-developed programs to test the system throughput with TCP and UDP protocols.

Although the Gbps demonstration system can provide over 1 Gbps data throughput at the wireless PHY layer, the achieved throughput above is limited due to the limitation of GE data interface itself, the performance of PC, and the overhead of Ethernet/IP protocols.

\section{Conclusions}

The presented optimal design rule and sensitivity analysis for LOS-MIMO antenna array give theoretical support for LOSMIMO system design, and it eases the choice of array shape by moving the limitation of perpendicular arrangement from earlier work.

Under the guide of derived antenna array design rule, a MIMO-OFDM-based high capacity radio system for 
TABLE 4: SNR/BER for different locations.

\begin{tabular}{|c|c|c|c|c|c|c|c|}
\hline Position & $T_{x} \log \mathrm{o}$ & $R_{x} \operatorname{logo}$ & Path number & 2nd path delay (ns) & 2nd path power $(\mathrm{dBc})$ & SNR $(\mathrm{dB})$ & BER \\
\hline 0 & 15.1 .3 & 0.4 .3 & 2 & 391 & -23 & 23.4 & $5 e-4$ \\
\hline 1 & 15.0 .3 & 0.4 .3 & 1 & - & - & 26.5 & 0 \\
\hline 2 & 14.5 .3 & 0.5 .3 & 2 & 439 & -26 & 26.5 & 0 \\
\hline 3 & 14.5 .3 & 0.5 .2 & 2 & 415 & -24.8 & 23.8 & $5 e-6$ \\
\hline 4 & 14.5 .3 & 0.5 .4 & 2 & 342 & -25.1 & 22 & $1 e-4$ \\
\hline 5 & 14.5 .4 & 0.5 .4 & 2 & 415 & -20.4 & 20 & $5 e-4$ \\
\hline 6 & 14.5 .4 & 0.5 .3 & 2 & 366 & -25.2 & 26 & 0 \\
\hline
\end{tabular}

TABLE 5: Throughput test results.

\begin{tabular}{lc}
\hline Application & Throughput (Mbps) \\
\hline Netperf & 860 \\
UDP & 880 \\
TCP & 760 \\
\hline
\end{tabular}

LOS channel at $15 \mathrm{GHz}$ frequency band is proposed with symmetrical square array at both $T_{x}$ and $R_{x}$. A GE switchbased SDR platform is designed to implement and verify the proposed system. Due to the good scalability and flexibility of GE interface, this platform has both the real-time processing capability in HAU and adaptability and programmability in GPP and hence is suitable for the development of future wireless communication systems.

On the GE SDR platform, the LOS-MIMO system yields throughput up to $1 \mathrm{Gbps}$ and spectral efficiency over $15 \mathrm{bps} / \mathrm{Hz}$ through field evaluation. The performance is supposed to be doubled with cross-polarization of antennas at the same array area. The proposed LOS-MIMO antenna array design and Gbps radio system are promising solutions to fixe broadband wireless access and wireless backhaul for the next generation networks.

\section{Acknowledgments}

This work was partially supported by National Basic Research Program of China (2012CB316000) and National S\&T Major Project (2012ZX03001026-003). This work was partially presented at CHINACOM 2010.

\section{References}

[1] N. Guo, R. C. Qiu, S. S. Mo, and K. Takahashi, "60$\mathrm{GHz}$ millimeter-wave radio: Principle, technology, and new results," EURASIP Journal on Wireless Communications and Networking, vol. 2007, Article ID 68253, 2007.

[2] R. Makri, P. Tsenes, D. Economou et al., "Next generation millimeter wave backhaul radio: overall system design for GbE $60 \mathrm{GHz}$ PtP wireless radio of high CMOS integration," in Proceedings of the 18th IEEE International Conference on Electronics, Circuits and Systems (ICECS '11), pp. 338-341, December 2011.

[3] C. F. Mecklenbrauker, M. Matthaiou, and M. Viberg, "Eigenbeam transmission over line-of-sight MIMO channels for fixed microwave links," in Proceedings of the International ITG
Workshop on Smart Antennas (WSA '11), pp. 1-5, February 2011.

[4] M. Shafi, M. Zhang, A. L. Moustakas et al., "Polarized MIMO channels in 3-D: models, measurements and mutual information," IEEE Journal on Selected Areas in Communications, vol. 24, no. 3, pp. 514-526, 2006.

[5] E. Torkildson, U. Madhow, and M. Rodwell, "Indoor millimeter wave MIMO: feasibility and performance," IEEE Transaction on Wireless Communications, vol. 10, no. 12, pp. 4150-4160, 2011.

[6] H. Zhang, S. Venkateswaran, and U. Madhow, "Channel modeling and MIMO capacity for outdoor millimeter wave links," in Proceedings of the IEEE Wireless Communications and Networking Conference (WCNC '10), pp. 1-6, April 2010.

[7] C. Sheldon, E. Torkildson, M. Seo, C. P. Yue, U. Madhow, and M. Rodwell, "A $60 \mathrm{GHz}$ line-of-sight $2 \times 2$ MIMO link operating at $1.2 \mathrm{Gbps}$," in Proceedings of the IEEE International Symposium on Antennas and Propagation and USNC/URSI National Radio Science Meeting (APSURSI '08), pp. 1-4, July 2008.

[8] F. Bøhagen, P. Orten, and G. E. Oien, "Design of optimal high-rank line-of-sight MIMO channels," IEEE Transactions on Wireless Communications, vol. 6, no. 4, pp. 1420-1424, 2007.

[9] F. Bøhagen, P. Orten, and G. E. Oien, "Modeling and analysis of a $40 \mathrm{GHz}$ MIMO system for fixed wireless access," in Proceedings of the IEEE 61st Vehicular Technology Conference (VTC '05), pp. 1691-1695, June 2005.

[10] P. Larsson, E. Res, and S. Kista, "Lattice array receiver and sender for spatially orthoNormal MIMO communication," in Proceedings of the 61st IEEE Vehicular Technology Conference (VTC '05), pp. 192-196, June 2005.

[11] L. Liu, W. Hong, H. Wang et al., "Characterization of lineof-sight MIMO channel for fixed wireless communications," IEEE Antennas and Wireless Propagation Letters, vol. 6, pp. 3639, 2007.

[12] J. Mitola, “The software radio architecture," IEEE Communications Magazine, pp. 26-38, 1995.

[13] K. Tan, J. Zhang, J. Fang et al., "Sora: high performance software radio using general purpose multi-core processors," in Proceedings of the 6th USENIX Symposium on Networked Systems Design \& Implementation (USENIX '9), pp. 99-107, 2009.

[14] J. Chen, Q. Wang, Z. Zhu, and Y. Lin, "An efficient software radio framework for WiMAX physical layer on cell multicore platform," in Proceedings of the IEEE International Conference on Communications (ICC '09), pp. 1-4, 2009.

[15] H. Guo, J. Zhao, J. Chen, X. Chen, and J. Wang, "High performance turbo decoder on CELL BE for WiMAX system," 
in Proceedings of the International Conference on Wireless Communications and Signal Processing (WCSP '09), pp. 1-5, Nanjing, China, November 2009.

[16] E. Telatar, "Capacity of multi-antenna Gaussian channels," European Transactions on Telecommunications, vol. 10, no. 6, pp. 585-595, 1999.

[17] K. W. Park and Y. S. Cho, "An MIMO-OFDM technique for high-speed mobile channels," IEEE Communications Letters, vol. 9, no. 7, pp. 604-606, 2005.

[18] H. Yang, "A road to future broadband wireless access: MIMOOFDM-based air interface," IEEE Communications Magazine, vol. 43, no. 1, pp. 53-60, 2005.

[19] D. C. Chu, "Polyphase codes with good periodic correlation properties," IEEE Transactions on Information Theory, vol. 18, pp. 531-532, 1972. 

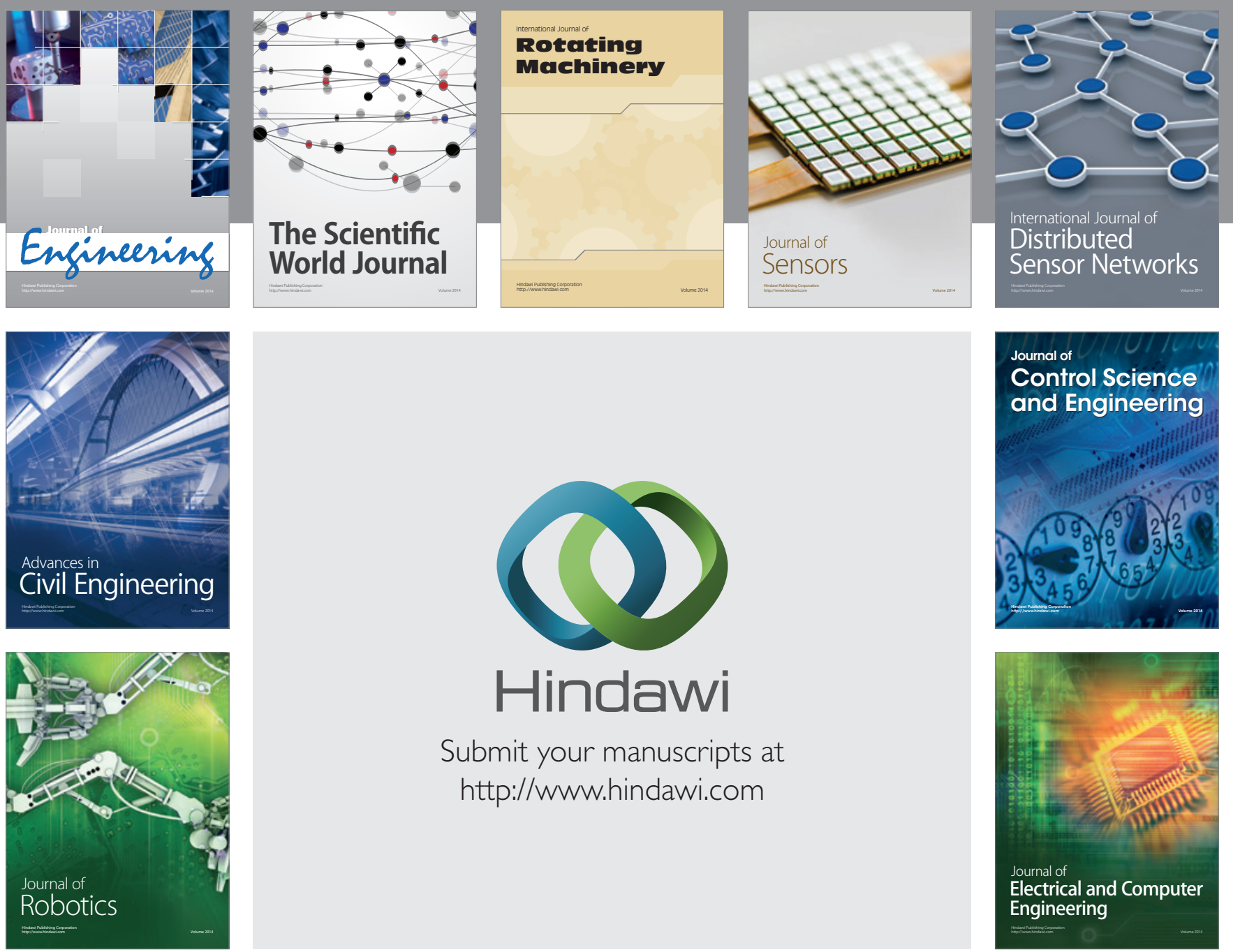

Submit your manuscripts at

http://www.hindawi.com
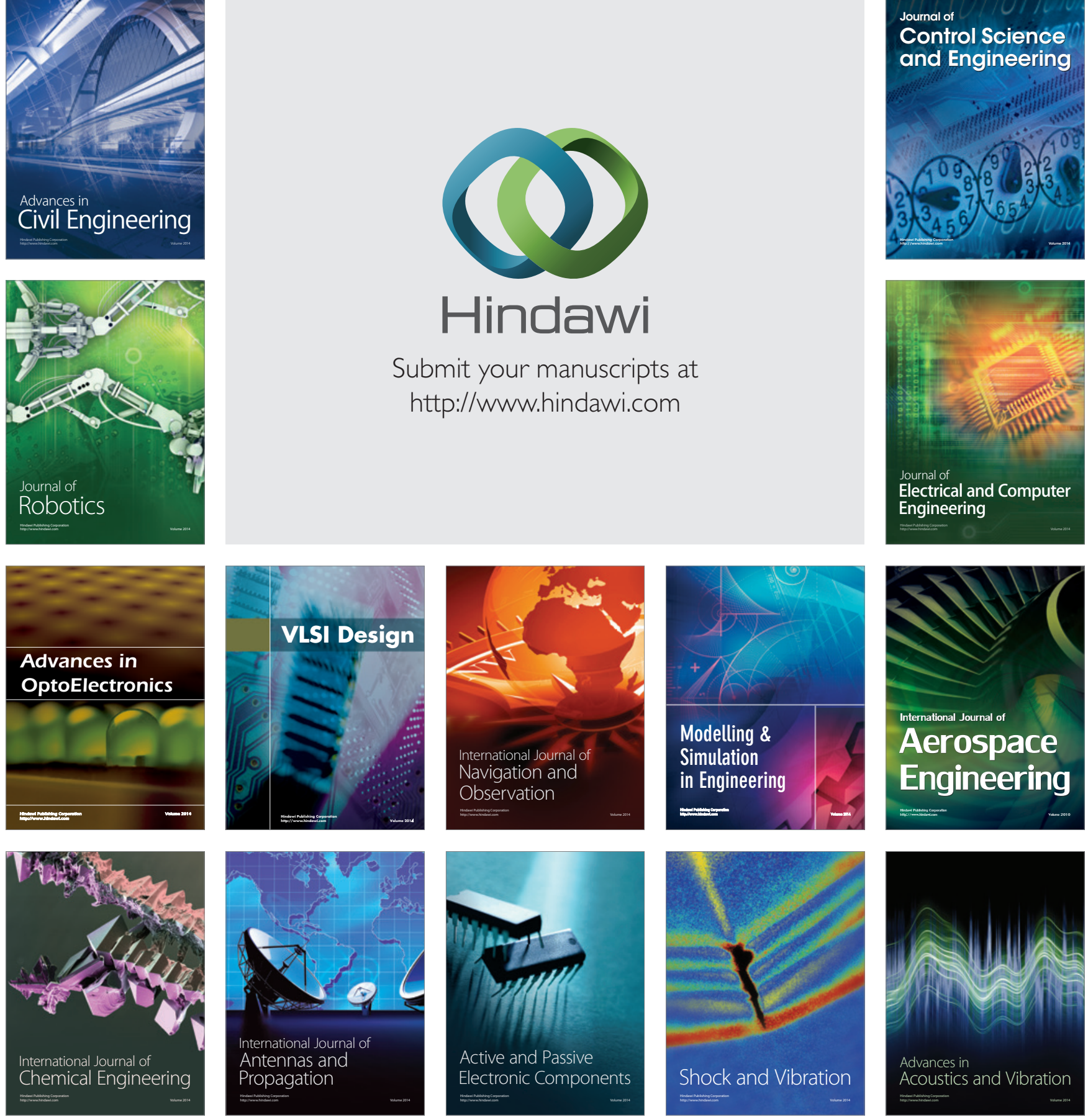